\title{
Optical Properties and Raman Scattering Investigation of Ag Doped ZnO Thin Film Prepared by Two-step Solution-based Deposition Method
}

\author{
A.N. Afaah ${ }^{1,2, a}$, N.A.M. Asib ${ }^{1,2, b}$, A. Aadila ${ }^{1,2, c}$, R. Mohamed $^{1,2, d}$, \\ K. A. Eswar ${ }^{1,2, e}$, M. Rusop ${ }^{1,3, f}$, Z. Khusaimi ${ }^{1,2, g}$ \\ ${ }^{1}$ NANO-SciTech Centre (NST), Institute of Science, Universiti Teknologi MARA (UiTM), 40450 \\ Shah Alam, Selangor, Malaysia \\ ${ }^{2}$ Faculty of Applied Sciences, Universiti Teknologi MARA (UiTM), 40450 Shah Alam, Selangor, \\ Malaysia \\ ${ }^{3}$ NANO-Electronic Centre (NET), Faculty of Electrical Engineering, Universiti Teknologi MARA \\ (UiTM), 40450 Shah Alam, Selangor, Malaysia

\footnotetext{
aafaahabdullah@yahoo.com, b amierahasib@yahoo.com, caadilaazizali@gmail.com druzianamohd@pahang.uitm.edu.my, ${ }^{\mathrm{d}}$ kevinalvin86@gmail.com, ${ }^{\mathrm{f}}$ nanouitm@gmail.com, gzurai142@salam.uitm.edu.my
}

Keywords: Ag; doping; Raman scattering; mist-atomization

\begin{abstract}
Mist-atomization deposition method was applied in order to grow $\mathrm{ZnO}$ nanoparticles on $\mathrm{Au}$-seeded glass substrates acting as seeded template. Ag doped $\mathrm{ZnO}$ thin films were deposited on $\mathrm{ZnO}$ seeded templates by solution-immersion method. The influence of Ag doping content on the optical and Raman scattering properties of $\mathrm{ZnO}$ films were systematically investigated by $\mathrm{UV}-\mathrm{Vis}$ transmittance measurement measured by ultra-violet visible spectroscopy (UV-Vis) and Raman scattering spectrum measured by Raman spectroscopy under room temperature. From UV-Vis transmittance measurement, the incorporation of $\mathrm{Ag}$ dopant to the $\mathrm{ZnO}$ makes the transmittance wavelength shifted to the shorter wavelength as compared to the pure $\mathrm{ZnO}$. From Raman spectra, 4 $\mathrm{cm}^{-1}$ downshift is observed in Ag-doped thin films as compared to pure $\mathrm{ZnO}$ thin films. This Raman peak shift shows that a tensile stress existed in the Ag-doped $\mathrm{ZnO}$ film.
\end{abstract}

\section{Introduction}

$\mathrm{ZnO}$ is a II-VI semiconductor compound with wide direct band-gap of $3.37 \mathrm{eV}$ and large excitation binding energy of $60 \mathrm{meV}$ at room temperature. In recent years, $\mathrm{ZnO}$ has been under extensive research because of its broad applications. $\mathrm{ZnO}$ also attracted many studies because of their capability and suitability for doping. Doped $\mathrm{ZnO}$ shows high electrical conductivity and improved optical properties by doping with suitable elements. Both stoichiometry and dopant impurities affect the electrical and optical characteristics of $\mathrm{ZnO}$ which make it either a near insulator or a semi-metal [1]. Usually $\mathrm{ZnO}$ is doped by $\mathrm{Cu}$ [2], $\mathrm{Al}$ [1], Sb [3], etc. Different technologies such as electron beam evaporation [1, 4], sol-gel spin coating [5], hydrothermal [6], ultrasonic spray pyrolysis [7, 8], thermal evaporation [9], RF magnetron sputtering [10], etc. have been reported to produce thin films of $\mathrm{Ag}$ doped $\mathrm{ZnO}$ with adequate performance for applications.

In order to optimize the optical and electrical properties, these techniques usually coupled with annealing of sample, during or after deposition process. Annealing procedures increase the optical transmittance value of the sample. The defects of the crystalline structures owned by $\mathrm{ZnO}$ i.e. O vacancies and $\mathrm{Zn}$ interstitial impurities can also be reduced by introducing this post-heat technique to the samples [4].

In this paper, we focus on the synthesis of un-doped and Ag-doped $\mathrm{ZnO}$ from solution-based method. We concentrate on the two-step deposition method; mist-atomization and solution immersion. The thin films then went a post-thermal treatment at $350,400,450$ and $500{ }^{\circ} \mathrm{C}$. The annealed samples were then compared to un-annealed thin films. The optical properties and Raman spectroscopic of as-prepared samples are studied. 


\section{Experimental}

Materials. The materials used in this work is zinc nitrate hexahydrate $\left(\mathrm{Zn}\left(\mathrm{NO}_{3}\right)_{2} \cdot 6 \mathrm{H}_{2} \mathrm{O}\right)$ Riedel-de Haën brand and stabilizer, hexamethylenetetramine (HMTA, $\mathrm{C}_{6} \mathrm{H}_{12} \mathrm{~N}_{4}$ ) of same brand, Riedel-de Haën with 99-100.5 \% pure analytical grade. Silver nitrate $\left(\mathrm{Ag}\left(\mathrm{NO}_{3}\right) \cdot 6 \mathrm{H}_{2} \mathrm{O}\right)$ is used as $\mathrm{Ag}$ source. Gold $(\mathrm{Au})$ target is used as the seeded catalyst of the glass substrate.

Synthesis. In this paper, the optical properties and Raman spectroscopic of un-doped $\mathrm{ZnO}$ and 1 at. $\%$ Ag-doped $\mathrm{ZnO}$ were investigated symmetrically. Six nanometer $(6 \mathrm{~nm})$ thick gold ( $\mathrm{Au}$ ) was coated on the glass substrates. From previous study, $6 \mathrm{~nm}$ found to be the most optimized thickness in synthesizing crystalline $\mathrm{ZnO}$ nanostructures [11]. Pure $\mathrm{ZnO}$ and Ag-doped $\mathrm{ZnO}$ were derived from an aqueous precursor solution prepared by combining $\mathrm{Zn}\left(\mathrm{NO}_{3}\right)_{2} \cdot 6 \mathrm{H}_{2} \mathrm{O}$ and HMTA with deionized water only for pure $\mathrm{ZnO}$, meanwhile for Ag-doped $\mathrm{ZnO}$, the starting materials were add up together with deionized water containing the appropriate amount of silver nitrate. The details of the experimental preparation are as follows. For the preparation of $\mathrm{Zn}^{2+}$ solution, $\mathrm{Zn}\left(\mathrm{NO}_{3}\right)_{2} \cdot 6 \mathrm{H}_{2} \mathrm{O}$ was dissolved in deionized water and stirred well to make sure it is homogenous. Then, HMTA solution is added to the $\mathrm{Zn}^{2+}$ solution. The ratio between $\mathrm{Zn}\left(\mathrm{NO}_{3}\right)_{2} \cdot 6 \mathrm{H}_{2} \mathrm{O}$ to HMTA is $1: 1$. The mixture was then stirred and heated on hot-plate stirrer at $60{ }^{\circ} \mathrm{C}$. After one hour of heating, the solution then aged for another 24 hours.

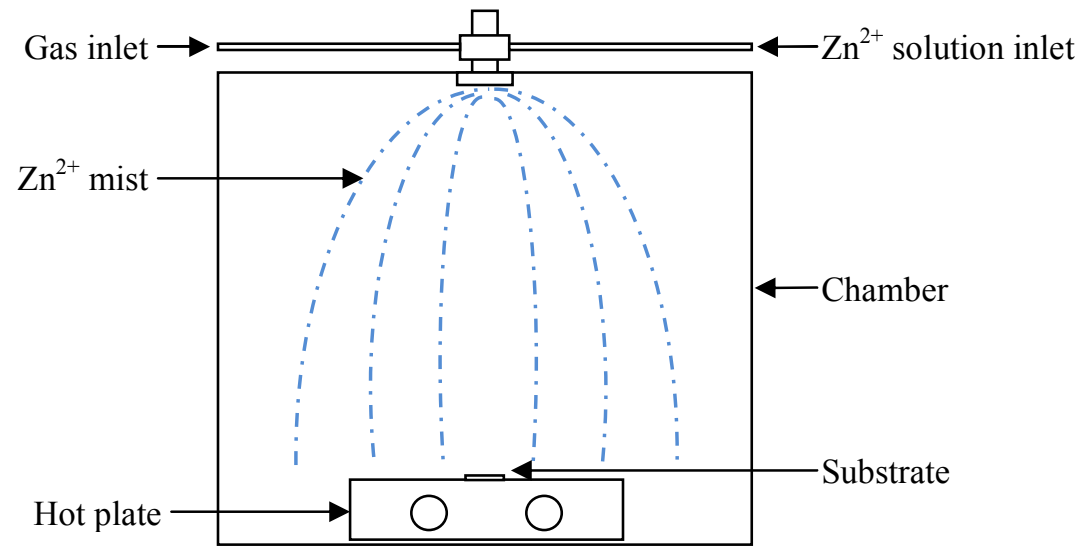

Fig. 1: Mist-atomizer chamber

Next, the solution is ready to be used for the first-deposition method; mist-atomization. The Auseeded glass substrates were placed on hot plate placed in the mist-atomizer chamber. The substrates were preheated at $250{ }^{\circ} \mathrm{C}$ for 10 minutes. Then, the $\mathrm{ZnO}$ solution was sprayed onto the glass substrates and left for 4 hours to make sure all the $\mathrm{ZnO}$ mists settled onto the substrates. The substrates then annealed at $450{ }^{\circ} \mathrm{C}$ for 1 hour to improve adhesion and remove possible impurities.

After that, the substrates undergo second deposition method; solution-immersion. The solution preparation for this method is the same as prepared for the first deposition method. The difference is $\mathrm{Ag}$ dopant is introduced to the mixture solution of $\mathrm{Zn}^{2+}$ and HMTA solution. For comparative study, no Ag dopant added for the un-doped $\mathrm{ZnO}$ solution. The substrates were placed in boiling tubes and un-doped and Ag-doped $\mathrm{ZnO}$ solution was poured into each boiling tubes. The boiling tubes needed to be sealed tightly for the formation of good crystalline $\mathrm{ZnO}$ nanostructures. Each sample was immersed in $90^{\circ} \mathrm{C}$ water bath for 4 hours.

The substrates then rinsed to remove any organic salts and prevent the contamination of the thin film. The glass substrates were annealed at different annealing temperatures of 350, 400, 450 and $500^{\circ} \mathrm{C}$. The samples were then compared to an un-annealed substrate.

Characterization. UV-Vis spectroscopy was performed using a Varian Cary $5000 \mathrm{UV}-\mathrm{Vis}$ spectrophotometer over the wavelength range between 300 and $900 \mathrm{~nm}$ with a data interval of $1 \mathrm{~nm}$. Raman spectra of the synthesized film were measured using Horiba Jobin Yvon-79 DU420A-OE325 Raman spectrophotometer with a He-Cd excitation laser source operating at $325 \mathrm{~nm}$. 


\section{Results and discussions}

Optical properties. The uv-vis transmission spectrum of pure $\mathrm{ZnO}$ thin film annealed at different temperatures is shown in Fig. 1.
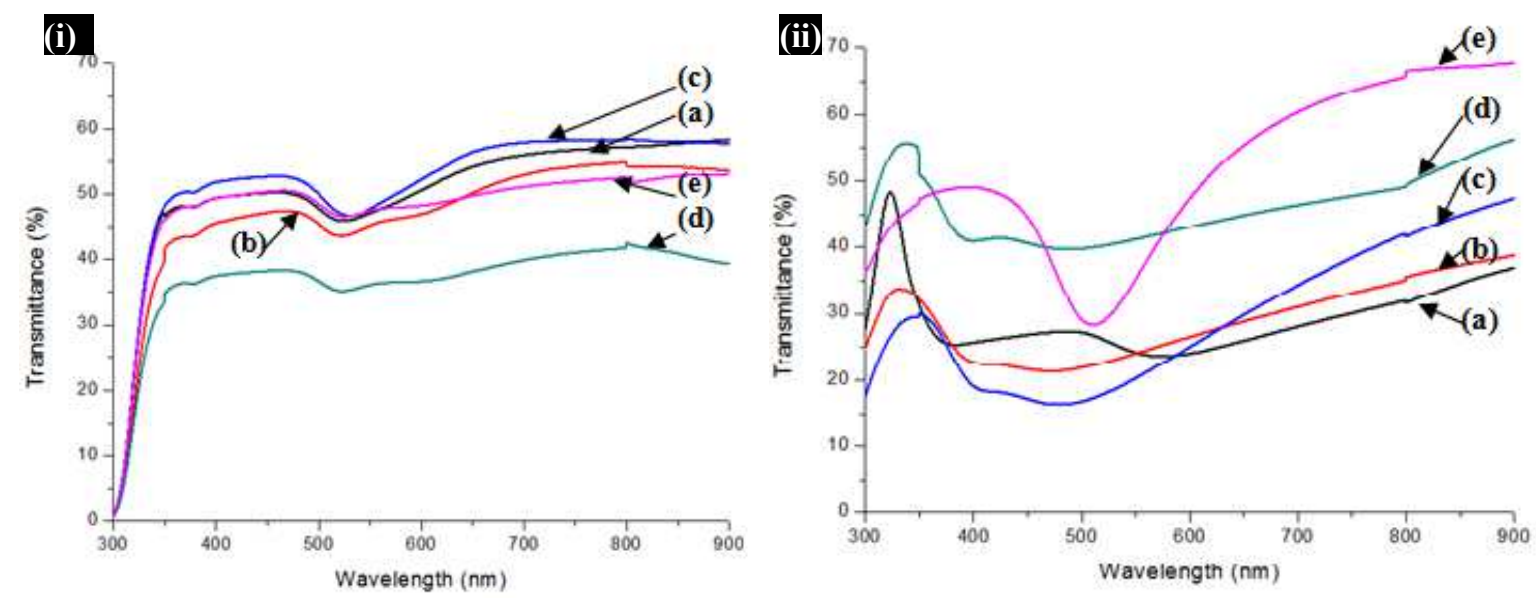

Fig. 2: UV-vis transmittance spectra of (i) pure $\mathrm{ZnO}$ annealed at (a) 0, (b) 350, (c) 400, (d) 450 and (e) $500{ }^{\circ} \mathrm{C}$ and (ii) Ag-doped $\mathrm{ZnO}$ annealed at (a) 0, (b) 350, (c) 400, (d) 450 and (e) $500{ }^{\circ} \mathrm{C}$

From Fig. 2(i), it can be seen that the transmission values of the film are low at short wavelengths; meanwhile at long wavelengths, the transmissions values are high. At short wavelengths, which is at the UV region, the pure $\mathrm{ZnO}$ exhibits good absorbance properties. The film acted as a dense material because of its high absorbing properties at UV region and as a transparent material at visible region. This condition is associated to the energy of the incident light [7]. When energies of photons are smaller than the band gap of $\mathrm{ZnO}$ film, they are insufficient for excitation of electrons from valence band to conduction band. Some of the photons have smaller energy compared to the band gap energy of $\mathrm{ZnO}$. The deficient of energy make them did not able to excite from valence band to the conduction band. These low energies remain at that certain level until some outer stimuli help to make some changes.

The natural defect of $\mathrm{ZnO}$ i.e. oxygen vacancies and interstitial $\mathrm{Zn}$ atoms, acted as donor impurities. These impurities may be ionized by these low energies. These impurities caused the film to have small absorbance and high transmittance values at visible region. From Fig. 2(i), it can be observed that at about 521-530 $\mathrm{nm}$ wavelength range, the transmittance values slightly increased as the wavelength increased. This range refers to the fundamental absorption region. Pure $\mathrm{ZnO}$ with $400{ }^{\circ} \mathrm{C}$ annealing temperature have the highest transmittance value amongst all samples. Meanwhile, $\mathrm{ZnO}$ thin film annealed at $450{ }^{\circ} \mathrm{C}$ have the lowest transmittance value.

From Fig. 2(ii), it shows that the Ag-doped sample annealed at $400{ }^{\circ} \mathrm{C}$ has the lowest transmittance value, meanwhile the sample annealed at $450{ }^{\circ} \mathrm{C}$ has the highest transmittance value. The incorporation of $\mathrm{Ag}$ dopant to the $\mathrm{ZnO}$ makes the transmittance wavelength shifted to the shorter wavelength as compared to the pure $\mathrm{ZnO}$ as in Fig. 2(i). From Fig. 2(ii), it can be observed that at about 360-400 $\mathrm{nm}$ wavelength range, the transmittance values slightly decreased as the wavelength increased. Meanwhile, the transmittance value of Ag-doped $\mathrm{ZnO}$ thin film annealed at $500{ }^{\circ} \mathrm{C}$ at $510 \mathrm{~nm}$ wavelength increased as the wavelength increased.

Raman studies. From the group theory, one will theoretically expect $A_{1}+2 B_{1}+E_{1}+2 E_{2}$, all will have two modes at the center of Brillouin zone [10]. The $\mathrm{A}_{1}, \mathrm{E}_{1}$ and $2 \mathrm{E}_{2}$ modes are Raman active. Only the two B1 modes are forbidden for Raman excitations. According to the selection rules we expect to observe the two $E_{2}$ modes and the $A_{1}$ (LO) mode in unpolarized Raman spectra taken in backscattering geometry. The frequencies of the fundamental optical modes in pure $\mathrm{ZnO}$ are $\mathrm{E}_{2}$ (low) is $101 \mathrm{~cm}^{-1}, \mathrm{E}_{2}$ (high) is $437 \mathrm{~cm}^{-1}, \mathrm{~A}_{1}$ (TO) is $380 \mathrm{~cm}^{-1}, \mathrm{~A}_{1}$ (LO) is $574 \mathrm{~cm}^{-1}, \mathrm{E}_{1}$ (TO) is 407 $\mathrm{cm}^{-1}$, and $\mathrm{E}_{1}(\mathrm{LO})$ is $583 \mathrm{~cm}^{-1}[12]$. 
Fig. 3(i) shows the Raman spectra of the undoped $\mathrm{ZnO}$ films with different annealing temperatures which are 0, 350, 400, 450 and $500{ }^{\circ} \mathrm{C}$. From Fig. 3(i), it is clear that the $\mathrm{A}_{1}$ (LO) mode and the $\mathrm{E}_{2}$ (high) mode of $\mathrm{ZnO}$ appear at about $577.9 \mathrm{~cm}^{-1}$ and $436.4 \mathrm{~cm}^{-1}$ for all the samples, respectively. Meanwhile, Fig. 3(ii) shows the Raman spectra of the 1 at. \% Ag-doped $\mathrm{ZnO}$ with different annealing temperatures of $0,350,400,450$ and $500{ }^{\circ} \mathrm{C}$. As shown in Fig. 3(ii), the $\mathrm{A}_{1}$ (TO) and $A_{1}$ (LO) modes were observed at 347.9 and $560 \mathrm{~cm}^{-1}$, respectively The $\mathrm{E}_{2}$ (high) mode appeared at about $433 \mathrm{~cm}^{-1}$.
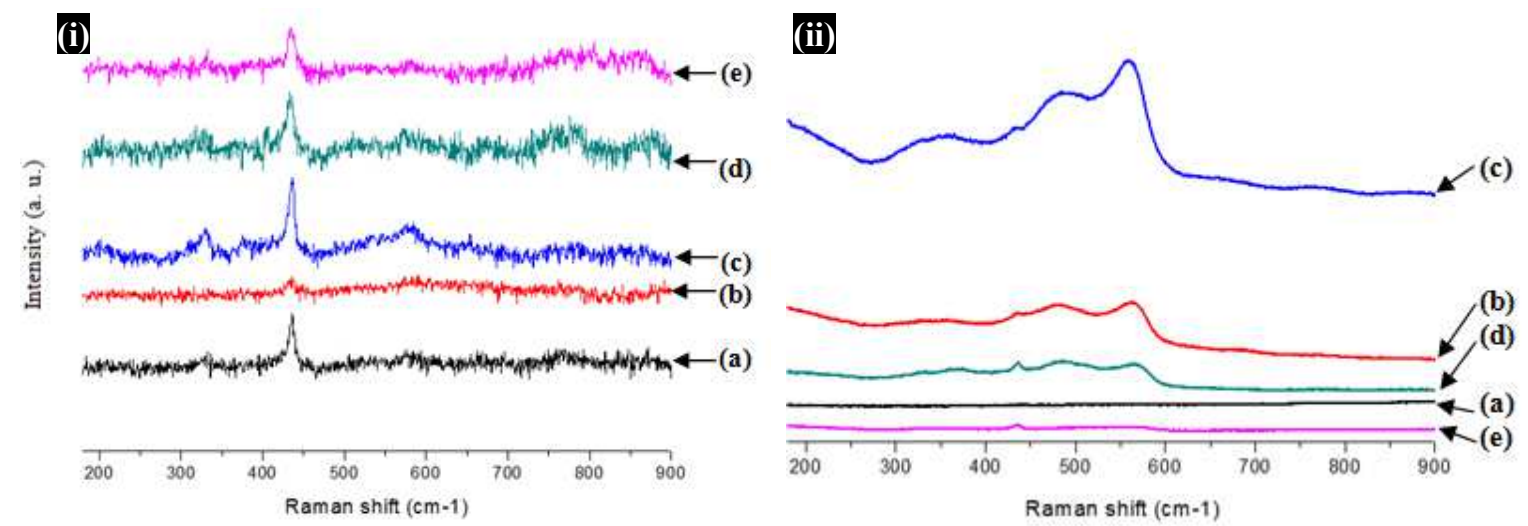

Fig. 3: Raman spectra of (i) pure $\mathrm{ZnO}$ annealed at (a) 0, (b) 350, (c) 400, (d) 450 and (e) $500{ }^{\circ} \mathrm{C}$ and (ii) $\mathrm{Ag}$-doped $\mathrm{ZnO}$ annealed at (a) 0 , (b) 350 , (c) 400 , (d) 450 and (e) $500{ }^{\circ} \mathrm{C}$

According to literature, the shift of the $\mathrm{E}_{2}$ mode can give the information on stress. The phonon frequency shifts related to the individual single crystal value that can be used to estimate remaining stress in the thin film [13]. Previous investigations have shown the relation between stress and $\mathrm{E}_{2}$ (high) mode: under a compressive stress the $\mathrm{E}_{2}$ (high) up shifts, where as a tensile stress will lead to its downshift $[10,12]$. Compared to the $\mathrm{E}_{2}$ (high) mode of the standard $\mathrm{ZnO}$ which is $437 \mathrm{~cm}^{-1}$, a downshift of $4 \mathrm{~cm}^{-1}$ was observed for the $\mathrm{E}_{2}$ (high) mode of the Ag doped $\mathrm{ZnO}$ film. This Raman peak shift shows that a tensile stress existed in the Ag doped $\mathrm{ZnO}$ film. The $\mathrm{A}_{1}$ (LO) mode with very high intensity was also observed in the Raman spectra of the Ag doped $\mathrm{ZnO}$ samples. The $\mathrm{A}_{1}$ (LO) mode is known to be related to the defects such as oxygen vacancy and $\mathrm{Zn}$ interstitial in $\mathrm{ZnO}$.

The high-frequency $E_{2}$ mode at 436.4 and $433 \mathrm{~cm}^{-1}$ is associated with vibration of the oxygen atoms [13]. Generally, the phonons of $E_{2}$ symmetry have two frequencies $E_{2}$ (high) and $E_{2}$ (low). $E_{2}$ (high) is related with the oxygen atom and $\mathrm{E}_{2}$ (low) related with the $\mathrm{Zn}$ sub lattice $[14,15]$. The incorporation of impurities, in this study is $\mathrm{Ag}$ dopant in the host lattice can introduce additional local vibrational modes (LVMs) in the Raman spectra. A possible physical mechanism for explaining LVMs is that defects induced by impurities break the translational symmetry of the crystal, thus relaxing the conservation of the wave vector and leading to the scattering of phonons with the wave vectors far from the Brillouin zone center [12]. Compared with undoped $\mathrm{ZnO}$ films, the LVM at about $485 \mathrm{~cm}^{-1}$ can be clearly observed in the patterns of $\mathrm{ZnO}$ :Ag films, such as shown in Fig. 3(ii).

\section{Conclusion}

As-prepared $\mathrm{ZnO}$ and $\mathrm{Ag}$-doped $\mathrm{ZnO}$ have been deposited by two-step deposition methods; mistatomizer and solution-immersion. The solution processing methods offer an economical route to the preparation of pure and Ag-doped $\mathrm{ZnO}$ thin films. Apart from that, the amount of dopant can be controlled easily by this method. The Raman investigation shows that Ag-doped $\mathrm{ZnO}$ have a downshift of $4 \mathrm{~cm}^{-1}$ which is observed at $\mathrm{E}_{2}$ (high) mode of the Ag doped $\mathrm{ZnO}$ film. This Raman peak shift shows that a tensile stress existed in the Ag-doped $\mathrm{ZnO}$ film.

\section{Acknowledgment}

The authors would like to thank Universiti Teknologi MARA (UiTM) Malaysia, Research Management Institute (RMI) of UiTM and Ministry of Higher Education Malaysia (MOHE) for their financial support under FRGS project no. 600-RMI/FRGS 5/3 (18/2012). 


\section{References}

[1] S.-Y.L. D.R. Sahu, Jow-Lay Huang, Improved properties of Al-doped ZnO film by electron beam evaporation technique, Microelectronics Journal, 38 (2007) 245-250.

[2] T.G.K. V.S. Khomchenkoa, A.K. Savin, L.V. Zavyalova, N.N. Roshchina, V.E. Rodionov, O.S. Lytvyn, V.I. Kushnirenko, V.B. Khachatryan, J.A. Andraca-Adame, Fabrication and properties of $\mathrm{ZnO}: \mathrm{Cu}$ and $\mathrm{ZnO}: \mathrm{Ag}$ thin films, Superlattices and Microstructures 42 (2007) 94-98.

[3] L.C. Oleg Lupan, Luis K. Ono, Beatriz Roldan Cuenya, Guangyu Chai, Hani Khallaf, Sanghoon Park, and Alfons Schult, Synthesis and Characterization of Ag- or Sb-Doped ZnO Nanorods by a Facile Hydrothermal Route, J. Phys. Chem. , 114 (2010) 12401-12408.

[4] S.-Y.L. D.R. Sahu, Jow-Lay Huang, Deposition of Ag-based Al-doped ZnO multilayer coatings for the transparent conductive electrodes by electron beam evaporation, Solar Energy Materials \& Solar Cells, 91 (2007) 851-855.

[5] M.H.M. N. D. Md Sin, M. Rusop, Z. Zulkifli, Electrically Conductive Zinc Oxide (ZnO) Nanostructures Prepared By Sol-gel Spin-coating, ESciNano 2010, (2010).

[6] J.C. Xu, Yonggang Zhang, Yunyan Ma, Shiyu Qu, Yi Xu, Chengtian, Effect of silver ions on the structure of $\mathrm{ZnO}$ and photocatalytic performance of $\mathrm{Ag} / \mathrm{ZnO}$ composites, Applied Surface Science, 255 (2008) 1996-1999.

[7] B. Ergin, E. Ketenci, F. Atay, Characterization of $\mathrm{ZnO}$ films obtained by ultrasonic spray pyrolysis technique, International Journal of Hydrogen Energy, 34 (2009) 5249-5254.

[8] K.Y. Liu, Beifang Yan, Hongwei Fu, Zhengping Wen, Meiwang Chen, Youjun Zuo, Jian, Effect of Ag doping on the photoluminescence properties of $\mathrm{ZnO}$ films, Journal of Luminescence, 129 (2009) 969-972.

[9] S.C. H.J. Pandya, A.L. Vyas, Integration of $\mathrm{ZnO}$ nanostructures with MEMS for ethanol sensor, Sensors and Actuators B, 161 (2012) 923-928.

[10] W.J.K. Li, C. Y. Ruan, H. B. Qin, G. P. Huang, G. J. Yang, T. Y. Liang, W. W. Zhao, Y. H. Meng, X. D. Yu, P. Cui, Y. T. Fang, L., Electrical properties and Raman scattering investigation of Ag doped ZnO thin films, Solid State Communications, 152 (2012) 147-150.

[11] S.A. Z. Khusaimi, H. A. Rafaie, M. H. Mamat, N. Abdullah, S. Abdullah, and M. Rusop, Photoluminescence and Structural Properties of Gold-Assisted Zinc Oxide Nanorods, Malaysian Journal of Science (Special Edition), 28 (2009) 197-201.

[12] L.Z.H. L.N. Wang, H.Q.Zhang, Y.Qiu, Y.Lang, G.Q.Liu, J.Y.Ji, J.X.Ma, Z.W.Zhao, Studying the Raman spectra of Ag doped $\mathrm{ZnO}$ films grown by PLD, Materials Science in Semiconductor Processing, 14 (2011) 274-277.

[13] S.K.S.a.G.J. Exarhos, Raman Spectroscopic Investigation of $\mathrm{ZnO}$ and Doped ZnO Films, Nanoparticles and Bulk Material at Ambient and High Pressure, Solid State Phenomenon, 55 (1997) 32-37.

[14] P.R.a.J. Kim, Facile and fast synthesis of flower-like $\mathrm{ZnO}$ nanostructures, Materials Letters, 93 (2013) 52-55.

[15] S.S. N. Kamal Singha, Shyama Ratha, S. Annapoornia, Optical and room temperature sensing properties of highly oxygen deficient flower-like $\mathrm{ZnO}$ nanostructures, Applied Surface Science, 257 (2010) 1544-1549. 